\title{
Iterations of Multivariate Polynomials and Discrepancy of Pseudorandom Numbers
}

\author{
Jaime Gutierrez and Domingo Gomez-Perez \\ Department of Mathematics and Computing, \\ Faculty of Science, University of Cantabria, \\ Santander E-39071, Spain \\ e-mail: $\{$ jaime, domingo\}@matesco.unican.es
}

\begin{abstract}
In this paper we present an extension of a result in [2] about a discrepancy bound for sequences of s-tuples of successive nonlinear multiple recursive congruential pseudorandom numbers of higher orders. The key of this note is based on linear properties of the iterations of multivariate polynomials.
\end{abstract}

\section{Introduction}

The paper [2] studies the distribution of pseudorandom number generators defined by a recurrence congruence modulo a prime $p$ of the form

$$
u_{n+1} \equiv f\left(u_{n}, \ldots, u_{n-m+1}\right) \quad(\bmod p), \quad n=m-1, m, \ldots,
$$

with some initial values $u_{0}, \ldots, u_{m-1}$, where $f\left(X_{1}, \ldots, X_{m}\right)$ is a polynomial of $m$ variables over the field $\mathbb{F}_{p}$ of $p$ elements. These nonlinear congruential generators provide a very attractive alternative to linear congruential generators and, especially in the case $m=1$, have been extensively studied in the literature, see [1] for a survey.

When $m=1$, for sequences of the largest possible period $t=p$, a number of results about the distribution of the fractions $u_{n} / p$ in the interval $[0,1)$ and, more generally, about the distribution of the points

$$
\left(\frac{u_{n}}{p}, \cdots, \frac{u_{n+s-1}}{p}\right)
$$

in the $s$-dimensional unit cube $[0,1)^{s}$ have been obtained, see the recent series of papers $[3,5-8]$ for more details. In the paper [2], the same method for nonlinear generators of arbitrary order $m>1$ is presented. In particular, the paper [2] gives a nontrivial upper bound on exponential sums and the discrepancy of corresponding sequences for polynomials of total degree $d>1$ which have a dominating term (see Theorem 1 and Theorem 2 in that paper). As in [2], we say that a polynomial $f\left(X_{1}, \ldots, X_{m}\right) \in \mathbb{F}_{p}\left[X_{1}, \ldots, X_{m}\right]$ has a dominating term if it is of the form

$$
f\left(X_{1}, \ldots, X_{m}\right)=a_{d_{1} \ldots d_{m}} X_{1}^{d_{1}} \cdots X_{m}^{d_{m}}+\sum_{i_{1}=0}^{d_{1}-1} \cdots \sum_{i_{m}=0}^{d_{m}-1} a_{i_{1} \ldots i_{m}} X_{1}^{i_{1}} \cdots X_{m}^{i_{m}}
$$


with some integers $d_{1} \geq 1, d_{2} \geq 0, \ldots, d_{m} \geq 0$ and coefficients $a_{i_{1} \ldots i_{m}} \in \mathbb{F}_{p}$ with $a_{d_{1} \ldots d_{m}} \neq 0$. We denote by $\mathcal{D} \mathcal{T}$ the class of polynomials having a dominating term.

In this paper we extend Theorem 1 and Theorem 2 of [2] to a very large class of polynomials, including arbitrary polynomials of degree greater than one with respect to the variable $X_{m}$, that is, polynomials $f$ with $\operatorname{deg}_{X_{m}}(f)>1$. This question appears in [2] as an important open problem. This note is based on properties about composition of multivariate polynomials which could be of independent interest.

The paper is divided into three sections. In Section 2 we study the behaviour of the polynomials under composition. Then Section 3 we extend the result of [2]. Finally, in Section 4 we pose some open problems.

\section{Iterations of Multivariate Polynomials}

Let $\mathbb{K}$ be an arbitrary field and let $f$ be a polynomial in $\mathbb{K}\left[X_{1}, \ldots, X_{m}\right]$. As in the paper [2], we consider, for $k=1,2, \ldots$, the sequence of polynomials $f_{k}\left(X_{1}, \ldots, X_{m}\right) \in \mathbb{K}\left[X_{1}, \ldots, X_{m}\right]$ by the recurrence relation

$$
f_{k}\left(X_{1}, \ldots, X_{m}\right)=f\left(f_{k-1}\left(X_{1}, \ldots, X_{m}\right), \ldots, f_{k-m}\left(X_{1}, \ldots, X_{m}\right)\right),
$$

where $f_{k}\left(X_{1}, \ldots, X_{m}\right)=X_{1-k}$, for $k=-m+1, \ldots, 0$.

In this section we will give sufficient conditions for the polynomial $f$ such that the polynomial sequence $f_{k}, k=-m+1, \ldots$, is linearly independent. In order to prove this we can suppose, without loss of generality, that $\mathbb{K}$ is an algebraically closed field. A central tool to study this sequence of polynomials is the following ring homomorphism :

$$
\phi: \mathbb{K}\left[X_{1}, \ldots, X_{m}\right] \rightarrow \mathbb{K}\left[X_{1}, \ldots, X_{m}\right]
$$

defined as: $\phi\left(X_{1}\right)=f$ and $\phi\left(X_{k}\right)=X_{k-1}$, for $k=2, \ldots, m$.

Lemma 1. With the above notations, we have the following:

$-\phi^{j}\left(f_{k}\right)=f_{k+j}, \quad$ for $j>0$ and $k=-m+1, \ldots, 0,1,2, \ldots$

- The polynomial $f$ has degree greater than zero with respect to the variable

$X_{m}$ if and only if $\phi^{j}$ is an injective map, for every $j \geq 1$. In particular, the

$\left\{f_{r}, f_{r+1}, \ldots, f_{r+m-1}\right\}$ are algebraically independent, for all $r \geq-m+1$.

Proof. The proof of the first part it is trivial by the definition of the rinh homorphism $\phi$.

On the other hand, we have that $\phi$ is injective map if and only if its kernel is trivial, that is, $\phi$ is injective if and only if

$$
\left\{p \in \mathbb{K}\left[X_{1}, \ldots, X_{m}\right], \quad \phi(p)=0\right\}=\{0\} .
$$


If $p \in \mathbb{K}\left[X_{1}, \ldots, X_{m}\right]$, then $\phi(p)=p\left(f, X_{1}, \ldots, X_{m-1}\right)$; so $p=0$ if and only if $\left\{X_{m-1}, \ldots, X_{1}, f\right\}$ are algebraically independent. If $\operatorname{deg}_{X_{m}}(f)>0$ then $X_{m}$ is algebraically dependent over $\mathbb{K}\left(f, X_{1}, \ldots, X_{m-1}\right)$. Consequently $\left\{X_{m-1}, \ldots, X_{1}, f\right\}$ are algebraically independent over $\mathbb{I K}$ if and only if we have $\operatorname{deg}_{X_{m}}(f)>0$.

Finally, by the first part, we see that $\phi^{r+m}\left(X_{m-j}\right)=f_{r+j}$, for $j=0, \ldots, m-$ 1. Now, the claim follows by induction on $r$.

We say that a multivariate polynomial $f\left(X_{1}, \ldots, X_{m}\right) \in \mathbb{K}\left[X_{1}, \ldots, X_{m}\right]$ is quasi-linear in $X_{m}$ if it is of the form $f=a X_{m}+g$ where $0 \neq a \in \mathbb{K}$ and $g \in \mathbb{K}\left[X_{1}, \ldots, X_{m-1}\right]$. We denote by $\mathcal{N} \mathcal{L}$ the class of non quasi-linear in $X_{m}$ polynomials of degree greater than zero with respect to the variable $X_{m}$. So, the class $\mathcal{N} \mathcal{L}$ is the set of all polynomials except the polynomials which do not depend on $X_{m}$ and the quasi-linear polynomials.

Lemma 2. Let $f$ be an element of $\mathcal{N} \mathcal{L}$. Then any finite family of the polynomials $f_{k}, k=-m+1, \ldots, 0,1, \ldots$, is linearly independent.

Proof. We prove it by induction on $m$. For $m=1$ it is obvious, because the degree is multiplicative with respect to polynomial composition. Now, we assume that $\operatorname{deg}_{X_{m}}(f)>0$ and we suppose that we have a nonzero linear combination:

$$
a_{r} f_{r}+a_{r+1} f_{r+1}+\cdots+a_{r+s} f_{r+s}=0,
$$

where $a_{j} \in \mathbb{K}$ and $a_{r} \neq 0$. We claim that $X_{m} \in \mathcal{I}$, where $\mathcal{I}$ is the ideal in the polynomial ring $\mathbb{K}\left[X_{1}, \ldots, X_{m}\right]$, generated by:

$$
\mathcal{I}=\left(X_{1}, \ldots, X_{m-1}, \bar{f}\right)
$$

with $\bar{f}=f-f(0, \ldots, 0)$.

By Lemma $1, \phi^{r+m-1}$ is an injective map and

$$
\phi^{r+m-1}\left(f_{-m+1}\right)=\phi^{r+m-1}\left(X_{m}\right)=f_{r} .
$$

Applying the inverse of $\phi^{r+m-1}$ to equation (3), we obtain:

$$
a_{r} X_{m}+a_{r+1} X_{m-1}+\cdots+a_{r+s} f_{s-m+1}=0 .
$$

We show that $\bar{f}_{t}=f_{t}-f_{t}^{0} \in \mathcal{I}$, where $f_{t}^{0}=f_{t}(0, \ldots, 0)$. By the uniqueness of the classical euclidean division

$$
f=\left(X_{1}-f_{t-1}^{0}\right) g_{1}+r_{1}\left(X_{2}, \ldots, X_{m}\right)
$$

and

$$
r_{1}\left(X_{2}, \cdots, X_{m}\right)=\left(X_{2}-f_{t-2}^{0}\right) g_{2}+r_{2}\left(X_{3}, \ldots, X_{m}\right) .
$$

Now, by recurrence, we have:

$$
f=\left(X_{1}-f_{t-1}^{0}\right) g_{1}+\cdots+\left(X_{m-1}-f_{t-m+1}^{0}\right) g_{m-1}+\left(X_{m}-f_{t-m}^{0}\right) g_{m}+g_{0},
$$

where $g_{i} \in \mathbb{K}\left[X_{i}, \ldots, X_{m}\right], i=0, \ldots, m$. 
Since, $f_{t}=f\left(f_{t-1}, \ldots, f_{t-m}\right)$ we have that $g_{0}=f_{t}^{0}$. Now, by induction on $t$, we will show that $\bar{f}_{t} \in \mathcal{I}$, for $t>0$. In order to see that, we observe that

$$
\begin{gathered}
\left.f_{t}=f\left(f_{t-1}, \ldots, f_{t-m}\right)\right)= \\
\left.\left.=\bar{f}_{t-1} g_{1}\left(f_{t-1}, \ldots, f_{t-m}\right)\right)+\cdots+\bar{f}_{t-m} g_{m}\left(f_{t-1}, \ldots, f_{t-m}\right)\right)+g_{0} .
\end{gathered}
$$

Then, $\bar{f}_{t}=f_{t}-g_{0} \in \mathcal{I}$.

Using the equation (4), we have:

$$
a_{r} X_{m}=-a_{r}^{-1}\left(a_{r+1} X_{m-1}+\cdots+a_{r+s} f_{s-m+1}\right) .
$$

And have just proved that $X_{m} \in \mathcal{I}$. So, there exist polynomials $w_{i} \in \mathbb{K}\left[X_{1}, \ldots, X_{m}\right]$, $i=1, \ldots, m$, such that

$$
X_{m}=X_{1} w_{1}+\cdots+X_{m-1} w_{m-1}+\bar{f} w_{m},
$$

then $X_{m}=\bar{f}\left(0, \ldots, 0, X_{m}\right) w_{m}\left(0, \ldots, 0, X_{m}\right)$. As consequence, we can write $f$ as follows:

$$
f=X_{1} h_{1}+\cdots+X_{m-1} h_{m-1}+\alpha X_{m}+\beta
$$

where $h_{i} \in \mathbb{K}\left[X_{i}, \ldots, X_{m}\right],(i=1, \ldots, m-1), \alpha, \beta \in \mathbb{K}$ and $\alpha \neq 0$. Now, we consider the polynomial

$$
H=f\left(X_{1}, \ldots, X_{m-1}, Y\right)-f\left(X_{1}, \ldots, X_{m-1}, Z\right) \in \mathbb{K}\left[X_{1}, \ldots, X_{m-1}, Y, Z\right] .
$$

We claim there exists a zero $\left(\alpha_{0,1}, \ldots, \alpha_{0, m-1}, \beta_{0}, \gamma_{0}\right) \in \mathbb{K}^{m+1}$ of the polynomial $H$, with $\beta_{0} \neq \gamma_{0}$. In order to prove this last claim, we write the polynomial $f$ as univariate polynomial in the variable $X_{m}$ with coefficients $b_{j}$ in the polynomial ring $\mathbb{K}\left[X_{1}, \ldots, X_{m-1}\right]$, for $j=0, \ldots, s$, that is, $f=b_{s} X_{m}^{s}+\cdots+b_{1} X_{m}+b_{0}$, for $j=0, \ldots, s$ and $b_{s} \neq 0$. So,

$$
H=b_{s}\left(Y^{s}-Z^{s}\right)+\cdots+b_{1}(Y-Z) .
$$

If a such zero does not exist, then the zero set of $h$ coincides with the zero set of the polynomial $Y-Z$. Since $Y-Z$ is an irreducible polynomial in $\mathbb{K}\left[X_{1}, \ldots, X_{m-1}, Y, Z\right]$, then by the Nullstellensatz theorem, (see for instance [4] ) $H$ is a power of $Y-Z$, i.e., there exists a positive natural number $t$ such that $H=\gamma(Y-Z)^{t}$, where $0 \neq \gamma \in \mathbb{K}$. We have the following:

$$
b_{s}\left(Y^{s}-Z^{s}\right)+\cdots+b_{1}(Y-Z)=\gamma(Y-Z)^{t} .
$$

From this polynomial equality, we obtain that $s=t$. Since $\gamma(Y-Z)^{s}$ is a homogenous polynomial, then $b_{s}\left(Y^{s}-Z^{s}\right)=\gamma(Y-Z)^{s}$. Now, from (5), we get that $s=1$ and $f$ must be $b_{1} X_{m}+b_{0}$, that is, $f$ is a quasi-linear polynomial in $X_{m}$. By the assumption $f \in \mathcal{N} \mathcal{L}$, this is a contradiction.

Finally, we evaluate the left hand of the polynomial equality (4) in the point $P_{0}=\left(\alpha_{0,1}, \ldots, \alpha_{0, m-1}, \beta_{0}\right)$, we obtain:

$$
a_{r} \beta_{0}+\ldots+a_{r+m-1} \alpha_{0,1}+a_{r+m} f\left(P_{0}\right)+\cdots+a_{r+s} f_{r+s-m}\left(P_{0}\right)=0 .
$$


We also evaluate $(4)$ in the point $Q_{0}=\left(\alpha_{0,1}, \ldots, \alpha_{0, m-1}, \gamma_{0}\right)$ and we obtain:

$$
a_{r} \gamma_{0}+\cdots+a_{r+m-1} \alpha_{0,1}+a_{r+m} f\left(Q_{0}\right)+\cdots+a_{r+s} f_{r+s-m}\left(Q_{0}\right)=0 .
$$

We observe that $f_{k}\left(P_{0}\right)=f_{k}\left(Q_{0}\right)$ for all $k \geq 0$. Thus, subtracting the equation (7) from the equation (6), we get $a_{r}\left(\beta_{0}-\gamma_{0}\right)=0$. Again, this is a contradiction and, the result follows.

We can also extend the above result to another class of polynomials. We say that a multivariate polynomial $f\left(X_{1}, \ldots, X_{m}\right) \in \mathbb{K}\left[X_{1}, \ldots, X_{m}\right]$ of total degree $d$, has the dominating variable $X_{1}$ if it is of the form

$$
f=a_{d} X_{1}^{d}+a_{d-1} X_{1}^{d-1}+\cdots+a_{0}
$$

where $d>0$ and $a_{i} \in \mathbb{K}\left[X_{2}, \ldots, X_{m}\right]$, with $a_{d} \neq 0$. We denote by $\mathcal{D} \mathcal{V}$ the class of polynomials having the dominating variable $X_{1}$.

Lemma 3. With the above notations, for polynomial $f \in \mathcal{D} \mathcal{V}$ the total degree of the polynomial $f_{k}$ is $d^{k}, k=1,2, \ldots$. In particular, if $d>1$, any finite family of the polynomials $f_{k}, k=-m+1, \ldots, 0,1, \ldots$, is linearly independent.

Proof. We prove this statement by induction on $k$. For $k=1$ it is obvious.

Now we assume that $k \geq 2$. We have

$$
f_{k}=a_{d} f_{k-1}^{d}+a_{d-1}\left(f_{k-2}, \ldots, f_{k-(m-1)}\right) f_{k-1}^{d-1}+\cdots+a_{0}\left(f_{k-2}, \ldots, f_{k-(m-1)}\right)
$$

We remark that for all

$$
\operatorname{deg}\left(a_{d-i}\right) \leq i, \quad i=0, \ldots, d,
$$

because $\operatorname{deg} f=d$. Using the induction assumption we obtain

$$
\begin{aligned}
& \operatorname{deg}\left(a_{d-i}\left(f_{k-2}, \ldots, f_{k-(m-1)}\right) f_{k-1}^{d-i}\right) \\
& =\operatorname{deg}\left(a_{d-i}\left(f_{k-2}, \ldots, f_{k-(m-1)}\right)\right)+\operatorname{deg}\left(f_{k-1}^{d-i}\right) \leq i d^{k-2}+(d-i) d^{k-1}
\end{aligned}
$$

for all $i=1, \ldots, d$. On the other hand

$$
\operatorname{deg}\left(a_{d} f_{k-1}^{d}\right) \geq \operatorname{deg}\left(f_{k-1}^{d}\right)=d^{k}
$$

Finally, we observe that $d^{k}>i d^{k-2}+(d-i) d^{k-1}$ for all $i=1, \ldots, d$.

We have the following corollary:

Corollary 1. If $f$ is a polynomial in $\mathbb{K}\left[X_{1}, X_{2}\right]$ of total degree greater than one, then any finite family of the polynomials $f_{k}, k=-m+1, \ldots, 0,1, \ldots$, is linearly independent.

Proof. It is an immediate consequence of Lemmas 2 and 3 
We observe that any polynomial in the class $\mathcal{N} \mathcal{L}$ has total degree greater than one. On the other hand, if $f$ is a linear polynomial, the sequence $f_{k}, k=1, \ldots$, is obviously linearly dependent.

The following examples illustrate that we have three different classes of multivariate polynomial in $m$ variables. The polynomial $f=X_{1}^{2}+X_{2} X_{1}$ has dominating variable $X_{1}$, that is, $f \in \mathcal{D} \mathcal{V}$, but it has not a dominating term, $f \notin \mathcal{D} \mathcal{T}$. We also have, that $f$ is not a quasi-linear polynomial in $X_{2}$. Conversely, $g=X_{1} X_{2}+1 \in \mathcal{D} \mathcal{T} \cap \mathcal{N} \mathcal{L}$, but $f \notin \mathcal{D V}$. Finally, $h=X_{1}^{2}+X_{2} \in \mathcal{D} \mathcal{T} \cap \mathcal{D V}$, but $h \notin \mathcal{N} \mathcal{L}$.

\section{Discrepancy Bound}

We denote by $\mathcal{T}$ the union of the three classes $\mathcal{T}=\mathcal{D} \mathcal{V} \cup \mathcal{D} \mathcal{T} \cup \mathcal{N} \mathcal{L}$.

Following the proof of Theorem 1 in [2], we note that the only condition that they require is the statement of the above results. So, as a consequence of Lemma 2 and 3 and Corollary 1 we have Theorem 1 and Theorem 2 of [2] for polynomials $f\left(X_{1}, \ldots, X_{m}\right) \in \mathbb{F}_{p}\left[X_{1}, \ldots, X_{m}\right]$ with $f \in \mathcal{T}$ if $m>2$ and for any non-linear polynomial $f$ if $m=2$.

As in the paper [2], let the sequence $\left(u_{n}\right)$ generated by (1) be purely periodic with an arbitrary period $t \leq p^{m}$. For an integer vector $\mathbf{a}=\left(a_{0}, \ldots, a_{s-1}\right) \in Z^{s}$, we introduce the exponential sum

$$
S_{\mathbf{a}}(N)=\sum_{n=0}^{N-1} \mathbf{e}\left(\sum_{j=0}^{s-1} a_{j} u_{n+j}\right),
$$

where $\mathbf{e}(z)=\exp (2 \pi i z / p)$.

Theorem 1. Suppose that the sequence $\left(u_{n}\right)$, given by (1) generated by a polynomial $f\left(X_{1}, \ldots, X_{m}\right) \in \mathbb{F}_{p}\left[X_{1}, \ldots, X_{m}\right]$ of the total degree $d \geq 2$ is purely periodic with period $t$ and $t \geq N \geq 1$. If $m=2$ or $f \in \mathcal{T}$, then the bound

$$
\max _{\operatorname{gcd}\left(a_{0}, \ldots, a_{s-1}, p\right)=1}\left|S_{\mathbf{a}}(N)\right|=O\left(N^{1 / 2} p^{m / 2} \log ^{-1 / 2} p\right)
$$

holds, where the implied constant depends only on $d$ and $s$.

As in the paper [2], for a sequence of $N$ points

$$
\Gamma=\left(\gamma_{1, n}, \ldots, \gamma_{s, n}\right)_{n=1}^{N}
$$

of the half-open interval $[0,1)^{s}$, denote by $\Delta_{\Gamma}$ its discrepancy, that is,

$$
\Delta_{\Gamma}=\sup _{B \subseteq[0,1)^{s}}\left|\frac{T_{\Gamma}(B)}{N}-\right| B||,
$$

where $T_{\Gamma}(B)$ is the number of points of the sequence $\Gamma$ which hit the box

$$
B=\left[\alpha_{1}, \beta_{1}\right) \times \ldots \times\left[\alpha_{s}, \beta_{s}\right) \subseteq[0,1)^{s}
$$

and the supremun is taken over all such boxes.

Let $D_{s}(N)$ denote the discrepancy of the points (2) for $n=0, \ldots, N-1$. 
Theorem 2. Suppose that the sequence $\left(u_{n}\right)$, given by (1) generated by a polynomial $f\left(X_{1}, \ldots, X_{m}\right) \in \mathbb{F}_{p}\left[X_{1}, \ldots, X_{m}\right]$ of the total degree $d \geq 2$ is purely periodic with period $t$ and $t \geq N \geq 1$. If $m=2$ or $f \in \mathcal{T}$, then the bound

$$
D_{s}(N)=O\left(N^{1 / 2} p^{m / 2} \log ^{-1 / 2} p(\log \log p)^{s}\right)
$$

holds, where the implied constant depends only on $d$ and $s$.

In particular, Theorems 1 and 2 apply to any non-linear with respect to $X_{1}$ polynomial. Thus these are direct generalizations of the results of [5].

\section{Remarks}

We have extended the results of [2] to a very large class of polynomials, including multivariate polynomials $f$ such that $\operatorname{deg}_{X_{m}}(f)>1$. The only remain open problem is for a subclass of polynomials of the form $g\left(X_{1}, \ldots, X_{m-1}\right)+a X_{m}$, where $a \in \mathbb{K}^{\times}$.

On the other hand, it would be very interesting to extend these results to the case of generators defined by a list of $m$ polynomials of $\mathbb{F}_{p}\left[X_{1}, \ldots, X_{m}\right]$ :

$$
\mathbf{F}=\left(f_{1}\left(X_{1}, \ldots, X_{m}\right), \ldots, f_{m}\left(\left(X_{1}, \ldots, X_{m}\right)\right)\right.
$$

For each $i=1, \ldots, m$ we define the sequence of polynomials $f_{i}^{(k)}\left(X_{1}, \ldots, X_{m}\right) \in$ $\mathbb{F}_{p}\left[X_{1}, \ldots, X_{m}\right]$ by the recurrence relation

$$
f_{i}^{(0)}=f_{i}, \quad f_{i}^{(k)}\left(X_{1}, \ldots, X_{m}\right)=f_{i}^{(k-1)}\left(f_{1}, \ldots, f_{m}\right), \quad k=0,1, \ldots .
$$

So, for very $k$, we have the following list of $m$ multivariate polynomials:

$$
\mathbf{F}^{\mathbf{k}}=\left(f_{1}^{k}\left(X_{1}, \ldots, X_{m}\right), \ldots, f_{m}^{k}\left(X_{1}, \ldots, X_{m}\right)\right) .
$$

Now, the question is for what general families of polynomials $\mathbf{F}$, for any two numbers $r$ and $s$ with $0 \leq r<s$ the polynomials $f_{i}^{r}-f_{i}^{s}, i=1, \ldots, m$, are linearly independent.

\section{Acknowledgments}

This research is partially supported by the National Spanish project PB97-0346.

\section{References}

1. J. Eichenauer-Herrmann, E. Herrmann and S. Wegenkittl, A survey of quadratic and inversive congruential pseudorandom numbers, Lect. Notes in Statistics, Springer-Verlag, Berlin, 127 (1998), 66-97.

2. F. Griffin, H. Niederreiter and I. Shparlinski, On the distribution of nonlinear recursive congruential pseudorandom numbers of higher orders, Proc. the 13th Symp. on Appl. Algebra, Algebraic Algorithms, and Error-Correcting Codes, Hawaii, 1999, Lect. Notes in Comp. Sci., Springer-Verlag, Berlin, 1999, 1719 , 87-93. 
3. J. Gutierrez, H. Niederreiter and I. Shparlinski, On the multidimensional distribution of nonlinear congruential pseudorandom numbers in parts of the period, Monatsh. Math., 129, (2000) 31-36.

4. M. Nagata, Theory of commutative fields, Translations of Mathematical Monograph, vol. 125, Amer. Math. Soc., Providence, R.IU., 1993.

5. H. Niederreiter and I. Shparlinski, On the distribution and lattice structure of nonlinear congruential pseudorandom numbers, Finite Fields and Their Applications, 5 (1999), 246-253.

6. H. Niederreiter and I. Shparlinski, On the distribution of inversive congruential pseudorandom numbers modulo a prime power, Acta Arithm., 92, (2000), 89-98.

7. H. Niederreiter and I. Shparlinski, On the distribution of pseudorandom numbers and vectors generated by inversive methods, Appl. Algebra in Engin., Commun. and Computing, 10, (2000) 189-202.

8. H. Niederreiter and I. E. Shparlinski, 'On the distribution of inversive congruential pseudorandom numbers in parts of the period', Math. Comp. (to appear). 